\title{
Tunnel Diode Large-Signal Equivalent Circuit Study and the Solutions of Its Nonlinear Differential Equations
}

\author{
Sidney B. Geller and Paul A. Mantek
}

(October 31, 1961)

\begin{abstract}
A large-signal equivalent circuit for the Esaki or tunnel diode is presented that characterizes the dynamic and static response of this semiconductor device. Nonlinear differential equations are written on the model and a graphical-numerical solution technique is described. Analog computer solutions for the nonlinear equations are also discussed for various modes of operation.
\end{abstract}

\section{Introduction}

Most of the equivalent circuit models presented for the tunnel diode have been small-signal types. The small-signal operation of this semiconductor device can be correctly characterized by its incremental resistance at a fixed bias point and some associated reactive elements. However, the largesignal departures from this bias point which typify bistable switching and relaxation oscillations make the model inadqeuate. In digital systems the active circuit elements are typically required to operate over extended ranges of their voltage-current characteristics. This paper presents a large-signal equivalent circuit for the tunnel-diode (fig. 1). This circuit characterizes botb the static and dynamic responses of the device for all modes of operation. The network enclosed in the dashed box simulates the static V-I characteristic of the tunnel diode.

Nonlinear differential equations are produced from the model and a graphical-numerical solution technique is described that is applicable to driven or self-excited systems. The equations are readily solved by analog computer techniques, particularly when curve following is employed on a monotonic curve that is presented in this paper.

An example of the graphical-numerical solution of the nonlinear equations for the relaxation oscillation case is presented in detail. Some comparisons are made between the graphical and analog computer solutions of bistable switching and sinusoidal oscillation modes of operation.

\section{Tunnel Diode Large-Signal Equivalent Circuit and Solution Techniques}

The validity of the small-signal tunnel diode model $[1]^{1}$ has been extended over a large-signal range by incorporating the static terminal characteristics of the device into the model shown in figure 1 . The lead inductance, $L_{s}$, the series body resistance, $R_{s}$, and the barrier capacitance, $C$, are assumed to maintain constant values over the operating range of interest [2]. Measurement techniques for these elements are described in the literature [3].

\footnotetext{
1 Figures in brackets indicate the literature references at the end of this paper.
}

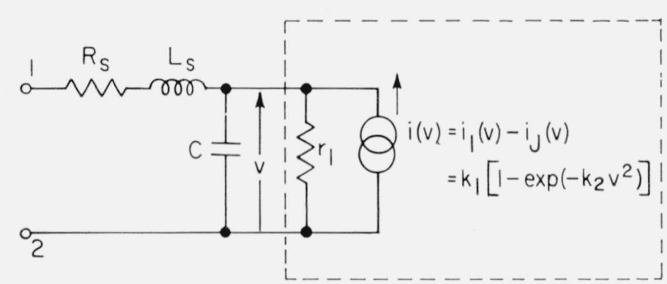

Figure 1. Large-signal tunnel diode equivalent circuit.

This study was made using the GE ZJ-56 (1 ma peak current) tunnel diodes. Typical values for $L_{s}, R_{s}$, and $C$ in this device are:

$$
\begin{aligned}
L_{s} & =6.0 \mathrm{nh} \\
R_{s} & =1.5 \Omega \\
C & =5.0 \mathrm{pf}
\end{aligned}
$$

Figure 2 shows a static V-I plot of the terminal characteristics of the ZJ-56 tunnel diode. Curve A in figure 3 is a broken-line approximation to this static plot which is simulated by the $i(v)$ current generator and resistance, $r_{1}$, of figure 1 (dashed box). The output from the $i(v)$ current generator (see appendix) is defined by either

$$
i(v)=i_{1}(v)-i_{j}(v) \quad(\text { fig. } 3 \text { curve B })
$$

or by an analytic approximation to curve B

$$
i(v)=k_{1}\left[1-\exp \left(-k_{2} v^{2}\right)\right] \quad \text { (fig. } 3 \text { curve C). }
$$

$i_{1}(v)$ is defined by the linear equation relating $v$ and $i$ in region 1 of curve $\mathrm{A} . \quad i_{j}(v)$ is defined by the linear equations relating $v$ and $i$ in each of the $j$ linear regions $(j=1,2,3,4)$ of curve $\mathrm{A}$. The resistance $r_{1}$ is set equal in value to the inverse slope of region 1 of curve $A$ in figure 3.

The following pair of equations can be written on the tunnel diode circuit in figure 4 with which all principal modes of operation such as switching and oscillating can be realized: 


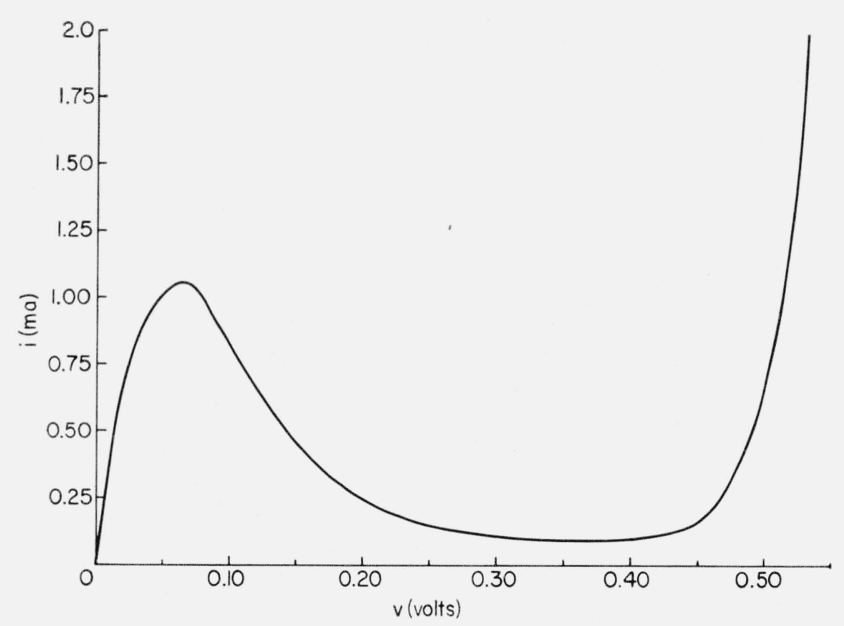

FIGURE 2. Static V-I characteristic for the GE ZJ-56 tunnel diode.

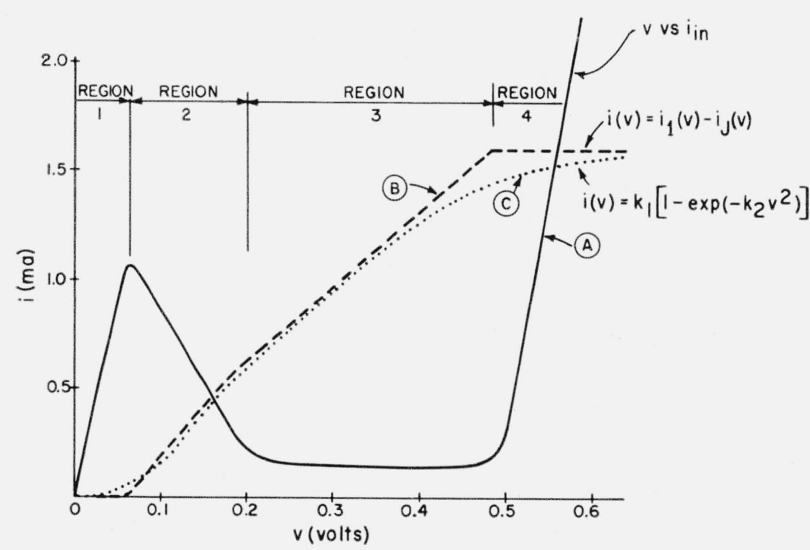

Figure 3. Curve A: Approximation to the GE ZJ-56 tunnel diode static V-I characteristic.

Curve B: Broken-line approximation to the current from the $\mathrm{i}(\mathrm{v})$ generator.

Curve C: Analytic approximation to the current from the $\mathrm{i}(\mathrm{v})$ generator.

$$
\left\{\begin{array}{l}
C \frac{d v}{d t}+\frac{v}{r_{1}}-k_{1}\left[1-\exp \left(-k_{2} v^{2}\right)\right]-i_{L}=0 \\
L \frac{d i_{L}}{d t}+R i_{L}=E(t)-v .
\end{array}\right.
$$

$E(t)$ is the applied voltage, $R=R_{x}+R_{s}$ and $L=L_{x}+L_{s}$.

Combining eqs (3) and (4) produces a nonlinear differential equation whose solution yields the junction voltage, $v$, i.e.,

$$
\begin{aligned}
\frac{d^{2} v}{d t^{2}}+\frac{1}{C} & {\left[\left(\frac{1}{r_{1}}+\frac{R C}{L}\right)-2 k_{1} k_{2} v \exp \left(-k_{2} v^{2}\right)\right] \frac{d v}{d t} } \\
& +\omega_{0}^{2}\left[\left(1+\frac{R}{r_{1}}\right) v-R k_{1}\left[1-\exp \left(-k_{2} v^{2}\right)\right]\right] \\
& =E(t) \omega_{0}^{2}
\end{aligned}
$$

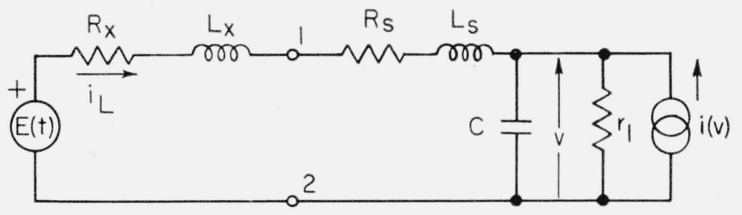

FIGURE 4. Tunnel diode circuit containing external $\mathrm{R}_{\mathbf{x}}$ and $\mathrm{L}_{\mathrm{x}}$ elements.

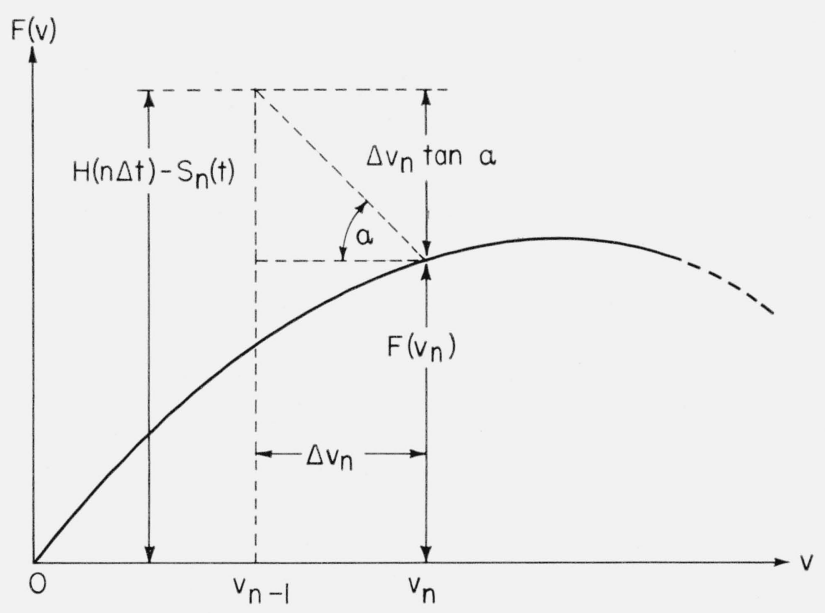

Figure 5. Typical construction increment in the $\mathrm{F}(\mathrm{v})$ versus v plane.

where $\omega_{0}^{2}=\frac{1}{L C}$. This equation does not lend itself to a closed form solution. It falls into the class of nonlinear equations of the type [4]

$$
\frac{d^{2} v}{d t^{2}}+f(v) \frac{d v}{d t}+g(v)=h(t) .
$$

After some investigation a graphical solution for this form was found and techniques for its application were worked out [5]. The solution process begins with an integration of eq (5) with respect to the time which yields

$$
\frac{d v}{d t}+F(v)+\int_{0}^{t} g(v) d t=H(t)+K
$$

where,

$$
\begin{aligned}
F(v) & =\frac{1}{C} \int_{0}^{v}\left[\left(\frac{1}{r_{1}}+\frac{R C}{L}\right)-2 k_{1} k_{2} v \exp \left(-k_{2} v^{2}\right)\right] d v \\
g(v) & =\omega_{0}^{2}\left[\left(1+\frac{R}{r_{1}}\right) v-R k_{1}\left[1-\exp \left(-k_{2} v^{2}\right)\right]\right] \\
H(t) & =\omega_{0}^{2} \int_{0}^{t} E(t) d t \\
K & =\text { integration constant. }
\end{aligned}
$$


Equation (7) is then converted into incremental form

$$
\frac{\Delta v_{n}}{\Delta t}+F\left(v_{n}\right)+S_{n}(t)=H(n \Delta t)-K
$$

where,

$$
S_{n}(t)=\int_{0}^{t} g[v(t)] d t
$$

A fixed value of $\Delta t$ is chosen heuristically and an angle

$$
\alpha=\arctan \frac{1}{\Delta t}
$$

is determined. It may be necessary to multiply equation (11) through by some constant in order to produce useful $\alpha$ 's. $\alpha$ is corrected to accommodate the departure of the abscissa-to-ordinate scaling of $F(v)$ versus $v$ from a one-to-one numerical relationship per unit length. Equation (11) is then rewritten into its final form preparatory to graphical solution as

$$
\Delta v \tan \alpha+F\left(v_{n}\right)=H(n \Delta t)+K-S_{n}(t) .
$$

The angle $\alpha$ is constructed physically on a template. Vertical lines of length $H(n \Delta t)-S_{n}(t)+K$ are constructed in the $F(v)$ versus $v$ plane as the solution proceeds. The successive $\Delta v_{n}$ 's are determined by the construction technique shown in the typical segment in figure 5. $S_{n}(t)$ is determined at each step of the process by applying the trapezoidal rule to the values of $g\left(v_{n}\right)$ and $g\left(v_{n+1}\right)$. This will be further detailed in the following section.

\section{Example of a Graphical-Numerical Solution of Equation (5), Relaxation Oscillation Case}

The circuit parameters were chosen to produce relaxation oscillations, i.e.,

$$
R<\frac{L}{|-r| C} \text { and } R<|-r|
$$

where $-r$ is the value of the inverse differential slope at each point in the negative resistance region of the V-I characteristic $(|-r| \approx 150 \Omega)$. A type GE ZJ-56 tunnel diode was employed in the circuit shown in figure 4 . The values of the parameters were

$$
\begin{aligned}
& R=20 \Omega \\
& L=100 \mu \mathrm{h} \\
& C=10 \mathrm{pf} \\
& E=0.25 \mathrm{v} \text { (step) } \\
& r_{1}=58.8 \Omega \\
& k_{1}=0.008 \\
& k_{2}=11.0 \\
& \omega_{0}^{2}=10^{15} \mathrm{rad} / \mathrm{sec} .
\end{aligned}
$$

These numerical values were put into eq (5) which was then integrated in respect to time. In order to produce angle $\alpha$ 's of reasonable size the equation was multiplied through by $30 \times 10^{-9}$. This yielded (in the form of equation (11))
$\Delta v_{n}\left(\frac{30 \times 10^{-9}}{\Delta t}\right)+30\left[1.7 v-.8\left[1-\exp \left(-11.0 v^{2}\right)\right]\right]$

$$
\begin{aligned}
& +30 \times 10^{6} \int_{0}^{t}\left[1.34 v-.16\left[1-\exp \left(-11.0 v^{2}\right)\right]\right] d t \\
& =7.5\left(10^{6}\right)(n \Delta t)+K .
\end{aligned}
$$

Since

$$
\Delta v_{n} \tan \alpha=\Delta v_{n}\left(\frac{30 \times 10^{-9}}{\Delta t}\right)
$$

therefore

$$
\alpha=\arctan \left(\frac{30 \times 10^{-9}}{\Delta t}\right)
$$

For example, if $\Delta t=30 \times 10^{-9}$ sec is chosen as the construction increment then $\alpha=45^{\circ}$. The $\Delta t$ chosen depends on the predicted response of the device. If $\Delta t$ is too large, the construction will be extremely coarse. If $\Delta t$ is too small, the solution will proceed very slowly. The value of $\Delta t$ may be easily altered during the solution process in order to achieve optimum speed and resolution. The analytical error between the exact solution of eq (7) and its graphical solution is of the order of $-1 / 2 \Delta^{2} v_{n}$ in each interval.

At this point the $F(v)$ versus $v$ and $g(v)$ versus $v$ plots were drawn where

$$
F(v)=30\left[1.7 v-.8\left[1-\exp \left(-11.0 v^{2}\right)\right]\right]
$$

and

$$
g(v)=30 \times 10^{6}\left[1.340 v-0.16\left[1-\exp \left(-11.0 v^{2}\right)\right]\right]
$$

Figures 6 and $7 \mathrm{~A}$ show these plots.

Values of $H(n \Delta t)=7.5\left(10^{6}\right)(n \Delta t) n=1,2,3, \ldots$ were tabulated.

Since the $\mathrm{F}(v)$ versus $v$ curve was found to be reasonably plotted on a 10:1 physical scale, the angle $\alpha$ was adjusted to compensate for this departure from a 1:1 relationship. This resulted in

$$
\begin{aligned}
& \alpha=5^{\circ} 44^{\prime} \text { for } \Delta t=30 \times 10^{-9} \mathrm{sec} \\
& \alpha=71^{\circ} 34^{\prime} \text { for } \Delta t=1 \times 10^{-9} \mathrm{sec} \\
& \alpha=85^{\circ} 15^{\prime} \text { for } \Delta t=0.3 \times 10^{-9} \mathrm{sec} .
\end{aligned}
$$

Three templates were constructed containing these angles.

The integration constant $K$, which is equal to $\dot{v}$ at $t=0$, was set equal to zero. The actual value of $K$ was sufficiently small to make this completely reasonable.

The $S_{n}(t)$ term is evaluated at each step of the graphical solution by the trapezoidal rule. Values of $v$ as a function of time such as $v(n \Delta t)$ and $v[(n+1) \Delta t]$ are determined by the successive constructions in the $F(v)$ versus $v$ plane. Then the values $g[v(n \Delta t)]$ and $g[v[(n+1) \Delta t]]$ are directly obtained from the $\mathrm{g}(v)$ versus $v$ plot. As the construction proceeds each quantity 


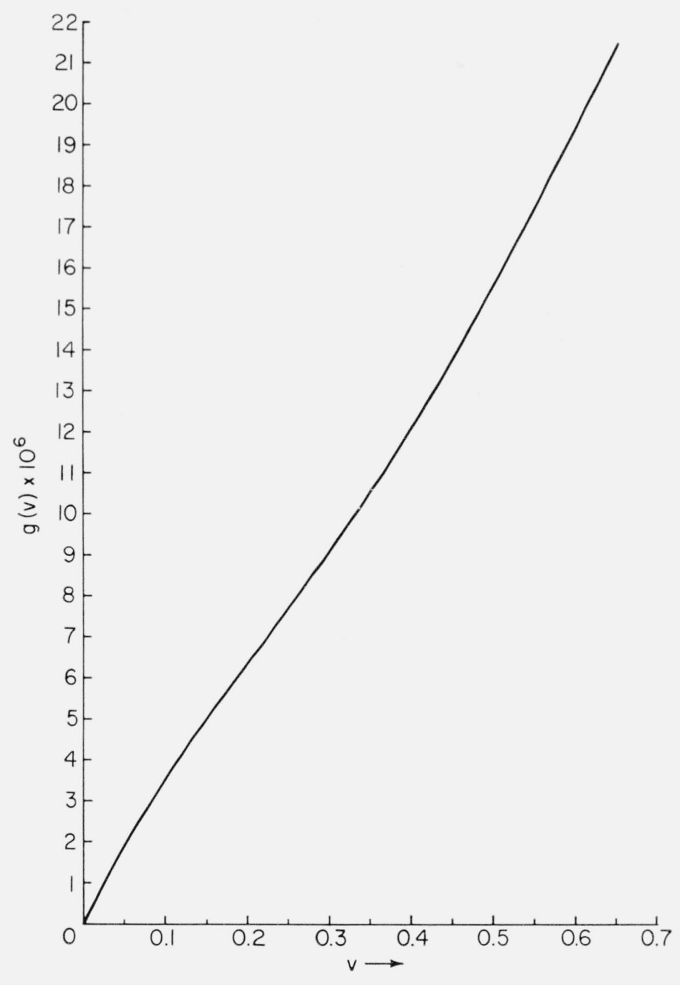

FIgURE 6. $\mathrm{g}(\mathrm{v})$ versus $\mathrm{v}$ plot employed in graphical solution of relaxation oscillator case.

$$
S_{n}(t)=\left(\frac{g(v[(n \Delta t)]+g(v[(n+1) \Delta t])}{2}\right) \Delta t
$$

is added on to the preceding total value of $S_{n}(t)$ and this new total is subtracted from the next applied $H(n \Delta t)$. This gives the length of the vertical construction line, $H(n \Delta t)-S_{n}(t)$ (as shown in fig. 5).

As each construction is made, the time required for each $\Delta v_{n}$ to occur is known from the relationship of $\Delta t$ to the angle $\alpha$ employed in the construction. When the $H(n \Delta t)-S_{n}(t)$ line falls below the $F(v)$ curve the construction reverses direction and proceeds towards the origin.

The angle $\alpha$ was changed during the construction process in order to produce maximum resolution and solution speed. For example, in the jump region the angle $\quad \alpha=85^{\circ} 15^{\prime} \quad$ (for $\Delta t=0.3 \times 10^{-9}$ sec) was employed.

Figure $7 \mathrm{~A}$ displays the $H(n \Delta t)-S_{n}(t)$ locus in the $F(v)$ versus $v$ plane for the relaxation oscillation case. Figure $7 \mathrm{~B}$ shows the junction voltage, $v$, versus time that was derived from the graphical construction. A single pulse from the actual ZJ-56 relaxation oscillator pulse train is shown superimposed upon a pulse produced by the graphical method which was based upon the analytic approximation of eq (2). The graphical process will produce a train of similar pulses if it is continued.

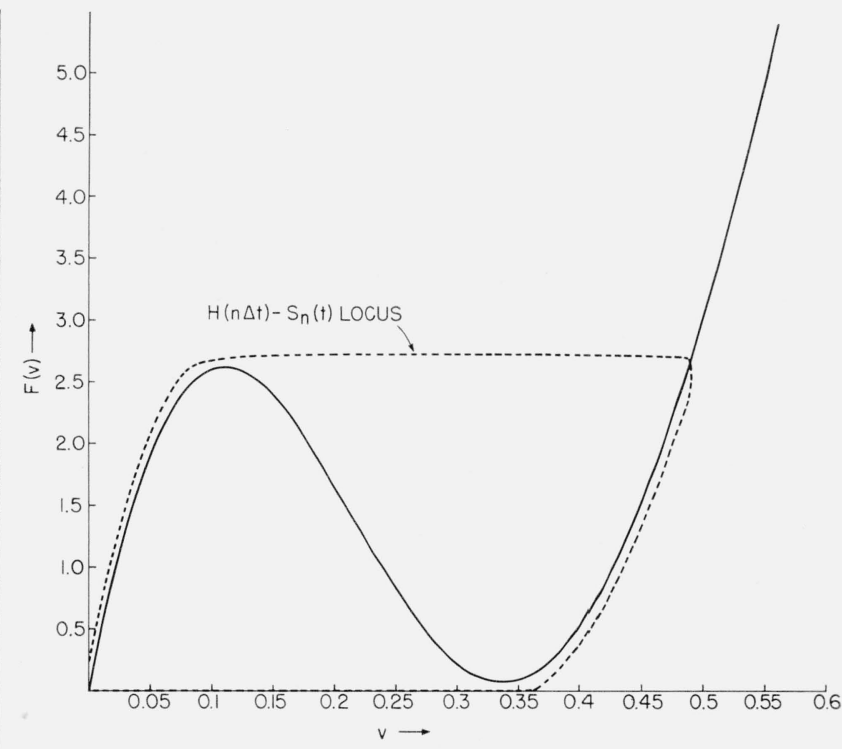

FIgURE 7. (A) $\mathrm{F}(\mathrm{v})$ versus $\mathrm{v}$ plot employed in the graphical solution of the relaxation oscillator case (solid line).

The dashed line is the locus of $H(n \Delta t)-S_{\mathrm{n}}(t)$ obtained during the graphical construction.

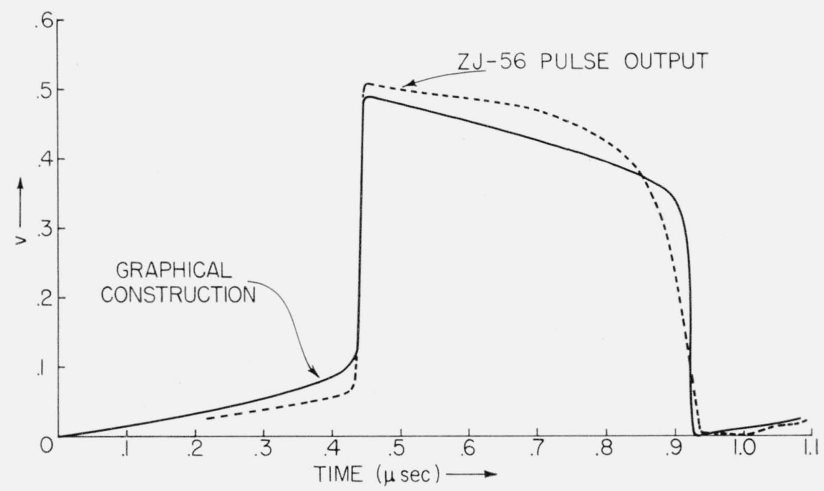

(B) Junction voltage, $\mathrm{v}$, versus time obtained from graphical solution (solid line) superimposed upon actual pulse (dashed line).

\section{Comparison of Analog and Graphical Results}

Equations (3) and (4) were mechanized on the National Bureau of Standards Mid-Century 500 Analog Computer employing curve-following techniques for both the broken-line and analytic $i(v)$ 's as defined by eqs (1) and (2). The method of constructing the broken-line $i(v)$ function is described in the appendix. Since these curves were relatively smooth and monotonic, (as compared to curve A, fig. 3) the curve-following operated well. The $R$, $L$, and $C$ elements were adjusted to produce bistable switching and (nearly) sinusoidal oscillations. Graphical solution technigues were then applied for these same operating conditions. Figure 8 displays the response of the system to a ramp driving function 


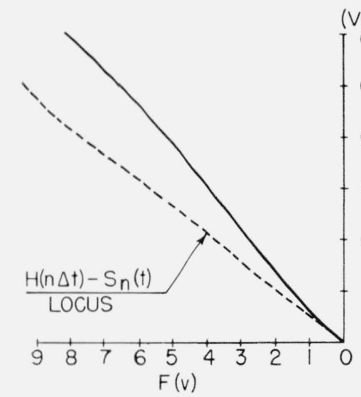

(A)

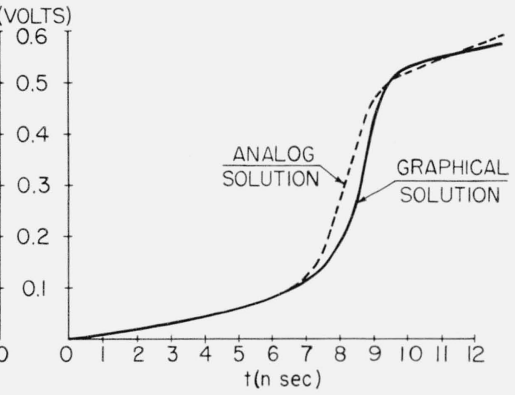

(B)

Figure 8. (A) Graphical construction in the $\mathrm{F}(\mathrm{v})$ versus v plane.

(B) Analog computer and graphical solution for junction $\mathrm{v}$ versus time (ramp-driven switching mode).

Figure 9. (A) Graphical construction in the $\mathrm{F}(\mathrm{v})$ versus $\mathrm{v}$ plane.

(B) Analog computer and graphical solution for junction v versus time (oscillatory mode).

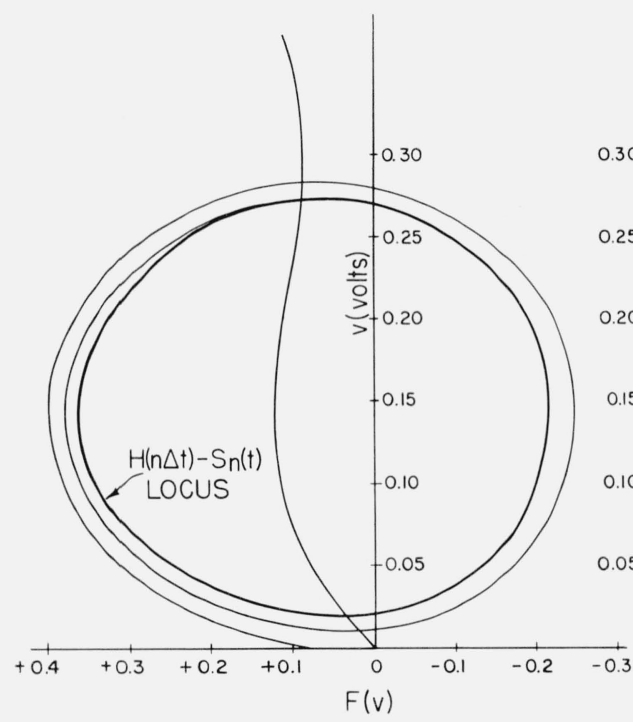

(A)

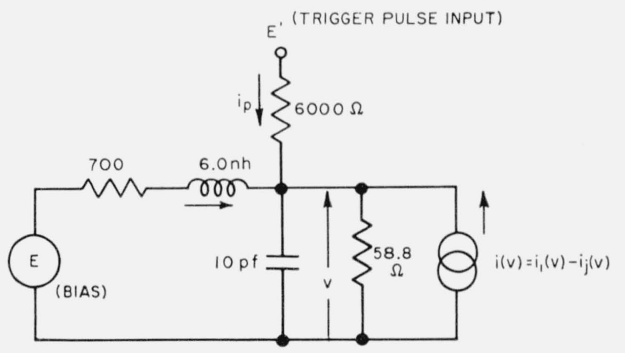

FIGURE 10. (A) Simulated bistable switching circuit with additional pulse triggering source.

(B) Analog computer mechanization for circuit in figure 10A.

with the circuit operating in the switching mode, i.e., $R>|-r|, R=470 \Omega, L=5.0 \mathrm{nh}$ and $C=2.0 \mathrm{pf}$. Figure 9 shows the sinusoidal response to a voltage step with $R<|-r|, R=1 \mathrm{c} \Omega, L=7.5$ nh and $C=2.0 \mathrm{pf}$.

The bistable switching case was simulated on the analog computer for another circuit configuration (fig. 10A). Here the tunnel diode was steadily biased with a voltage, $E$, that produced a stable singular point near the peak in region 1 (curve $\mathrm{A}$,

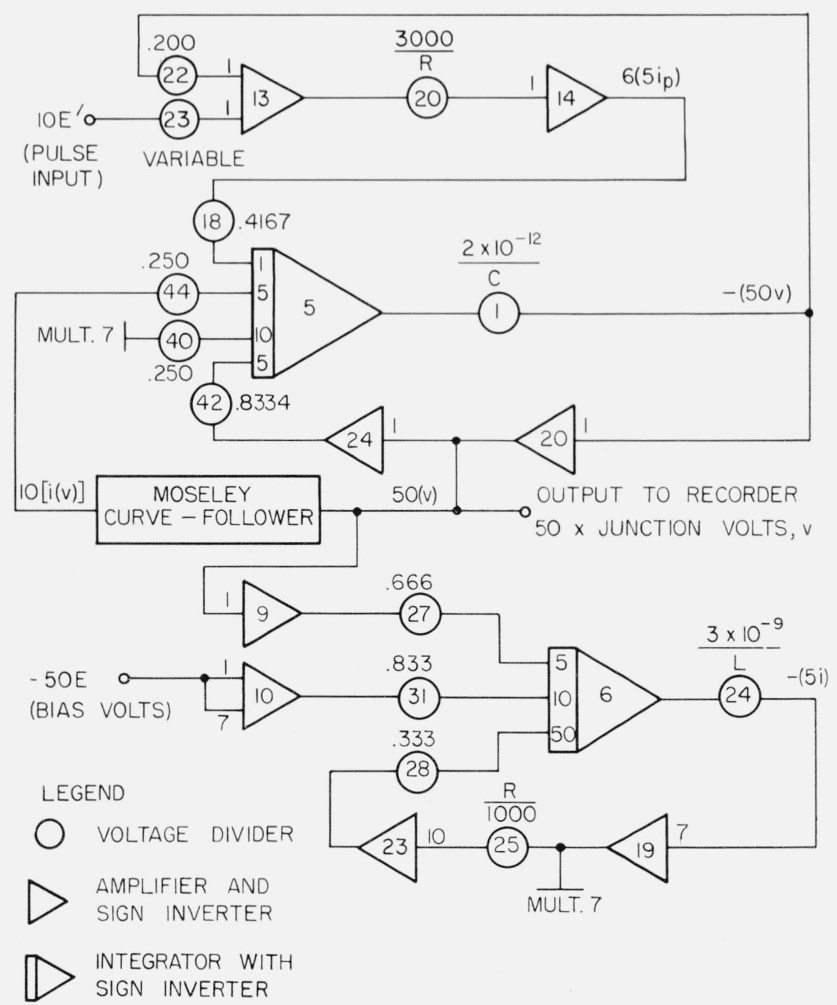


fig. 3). The circuit was then triggered over to the second stable singular point in region 4 by an injected current pulse, $i_{p}$. It was possible to observe the effects of pulses of different duration and amplitudes on the triggering process. This technique can be used to study the optimum trigger requirements for the tunnel diode and other negative resistance devices. Figure 10B shows the analog computer mechanization. The $i(v)$ function eq (1) was plotted with a conducting material and curve-followed during the simulation process.

\section{Appendix}

Crisson [6] has presented a method for producing a voltage-controlled negative resistance at the input terminals of an "ideal amplifier" with feedback. It can be shown that the network enclosed by the dashed line in figure 1 displays the same characteristics as the "ideal amplifier."

The "ideal amplifier" shown in figure $11 \mathrm{~A}$ can be represented by the circuit shown in figure 11B. The resistance, $r_{j}$, seen looking into the 1-2 terminals of the circuit is

$$
r_{j}=\frac{R_{1} R_{2}}{R_{1}+R_{2}-M_{j}}(j=1,2,3,4 .)
$$

where $M_{j}$ is a variable mutual impedance term. When $M_{j}>R_{1}+R_{2}$ then $r_{j}<0$ which produces a negative resistance at terminals $1-2 . \quad M_{j}$ can therefore be the basis for the simulation of the tunnel diode static V-I characteristic with its alternating positive and negative regions. Resistance $r_{1}$ (see fig. 1) is set equal to the inverse slope of region 1 in curve $\mathrm{A}$ figure 3. Therefore, if $M_{1}$ is set equal to zero in this region, then the parallel combination of $R_{1}$ and $R_{2}$ is chosen to be equal in value to $r_{1}$. In any one of the other three regions the variable $M_{j}$ can be be determined from

$$
M_{j}=\left(R_{1}+R_{2}\right)\left(1-\frac{r_{1}}{r_{j}}\right) .
$$

A conversion can be made from the circuit in figure $11 \mathrm{~B}$ to the circuit enclosed in the dashed box in figure 1 in which the current generator output is

$$
\begin{aligned}
\left.i(v)\right|_{j} & =i_{1}(v)\left(1-\frac{r_{1}}{r_{j}}\right) \\
& =i_{1}(v)-i_{j}(v)
\end{aligned}
$$

where, $i_{1}(v)$ and $i_{j}(v)$ are defined by the functional relationship of the current to the voltage in each linear region of curve $\mathrm{A}$ in figure 3 and each region is bounded by the breakpoints on the curve. Curve $\mathrm{B}$ in figure 3 is the monotonic $i(v)$ curve that is produced by eq (24). This curve is easily followed by curve-following analog techniques. An analytic approximation to curve $\mathrm{B}$ was also produced for use in obtaining a total differential equation for the junction voltage, $v$, of the tunnel diode. The approximation is
(A)

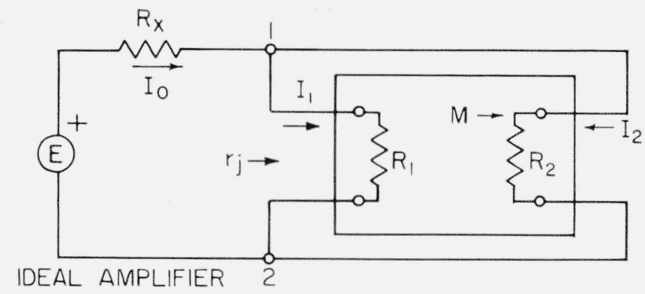

(B)

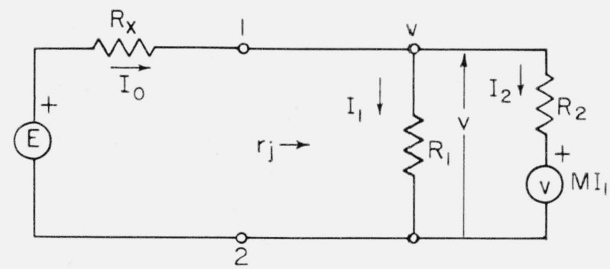

Figure 11. (A) Ideal amplifier.

(B) Network equivalent to the "ideal amplifier."

$$
i(v)=k_{1}\left[1-\exp \left(-k_{2} v^{2}\right)\right] \text { (fig. } 3 \text { curve C) }
$$

where, $k_{1}=0.008$ and $k_{2}=11.0$ for the GE ZJ-56 tunnel diode type that is used in this study.

The $i(v)$ curve B for use in analog simulation is constructed by differentiating eq (24) in respect to $v$ which gives

$$
\left.\frac{d i(v)}{d v}\right|_{j}=\frac{d i_{1}(v)}{d v}-\frac{d i_{j}(v)}{d v}
$$

For each of the $j$ linear regions $(j=1,2,3,4)$ of curve $\mathrm{B}$ a line with slope $\left.\frac{d i(v)}{d v}\right|_{j}$ is drawn from region breakpoint-to-breakpoint starting at the origin. Note that $\left.\frac{d i(v)}{d v}\right|_{j=1}=0$.

The authors thank E. S. Sherrard for his assistance with the analog computer mechanization.

\section{References}

[1] M.E. Hines, High-frequency negative-resistance circuit principles for Esaki diode applications, Bell System Tech. J. 39, 477 (1960).

[2] S. Cohen, Tunnel diode characterization, Elect. Equip. Eng. 8, 102 (1960).

[3] General Electric, Tunnel diode manual, (1961).

[4] S. Lefshetz, Differential equations: geometric theory, Interscience Publishers, 261 (1957).

[5] P. S. Hsia, A graphical analysis for nonlinear systems, Proc. IEE 99, 125 (1952).

[6] G. Crisson, Negative impedances in the twin 21 type repeater, Bell System Tech. J. 10, 485 (1931). 